\title{
LA VOLUNTAD DE SABER COMO PRÁCTICA DE LIBERACIÓN
}

\section{ÁNGELA MARÍA ESTRADA MESA ${ }^{1}$}

...Siempre he desconfiado un tanto del tema general de la liberación, en la medida en que, si no lo tratamos con algunas precauciones y en el interior de deteminados límites, se corre el riesgo de recurrir a la idea de que existe una naturaleza o un fondo humano que se ha visto enmascarado, alienado o aprisionado en y por mecanismos de represión como consecuencia de un deteminado número de procesos históricos, económicos y sociales.

Foucault, 1984

Resumen: La investigación de la cual este texto hace parte sigue el camino abierto por Judith Butler (1987, 1990ª 1990b), en el sentido de someter las teorías del postestructuralismo a una refomulación específicamente feminista antes que su mera aplicación a los problemas del género. Tal empeño, sin embargo, se enmarca en la transfomación que el formalismo postestructuralista ha experimentado al migrar hacia otros campos problemáticos, en los cuales "ha adquirido una vida nueva y transplantada en el terreno de la teoría cultural." (Butler, 1990a: 11).

Palabras clave: sujeto, subjetividad, corporalidad, cultura, mujer, identidad, lo femenino, género, filosofía feminista.

Abstract: The research of which this text makes part follows the road opened up by Judith Butler $\left(1987,1990^{a}, 1990 b\right)$, in the sense of subjecting the posts tructuralist theories to a specifically feminist reformulation instead of merely applying them to gender problems. Such an endeavor, however, is framed in the transformation that poststructuralist formalism has experienced by turning into other problematic fields, in which it "has acquired a new life and has been transplanted into the grounds of cultural theory." (Butler, 1990a: 11).

Key words: subject, subjectivity, corporality, culture, woman, identity, the feminine thing, gender, feminist philosophy.

\section{Introducción}

La puesta en cuestión de la precedencia del sujeto a todo conocimiento, ética o cultura, a la manera en que lo han teorizado la fenomenología o el existencialismo, ha sido confrontada por al menos dos diferentes tradiciones críticas. Tanto desde la perspectiva arqueológica hemenéutica de Foucault $(1976,1984)$ como desde el socioconstruccionismo de Gergen (MacNamee y

\footnotetext{
${ }^{1}$ Psicóloga, Magíster en Investigación y tecnología Educativas. Candidata a Doctorado en Filosofía. Prof esora Asociada. aestrada@uniandes.edu.co Departamento de Psicología Universidad de Los Andes
} 
Gergen, 1999) si el sujeto es previo al discurso, bastaría con 'hacer saltar los cerrojos represivos' para que el sujeto se reconciliase consigo mismo y con su naturaleza. En el mismo sentido, si el sujeto es anterior a la cultura y por lo tanto a la interacción social, no puede ser más que una categoría vacía. Así pues, el principal rendimiento de la historia de la cultura, de la filosofía y de las ciencias sociales y específicamente en lo que toca a las producciones discursivas sobre el sujeto, es el sujeto mismo, las distintas posiciones de sujeto cons tituidas a lo largo de la historia.

\section{La misoginia ilustrada y la función contemporánea de la filosofía}

Son principalmente dos características complejas las construidas por el pensamiento moderno e ilustrado sobre la mujer: una, caracterizada por un arquetipo de lo femenino que se opone a la individuación de la mujer, y otra, relacionada con el estatuto moral propuesto para ella.

El eterno femenino como arquetipo al que las mujeres se ads criben como foma de cumplir personalmente con una forma de identidad que no es personal. Desde la construcción de lo femenino en las diosas como personajes de la historia de las mujeres (Loraux, 1991), se llega a establecer no sólo el núcleo de la metonimia femenina con la maternidad (más propiamente con la matriz) y de es te modo con la naturaleza, sino también las características que se le atribuyen al modo de la existencia de las mujeres.

Inexorablemente todas las diosas y dioses remiten a una gran diosa -Gea- la Diosa Madre (La Tierra) cuyo poder es tan grande que debe ser conjurado. La Diosa constituye una noción abstracta muy general de lo femenino, una categoría en la cual todas las mortales deben encajar, sin necesidad alguna de individualizarse, sino, por el contrario, de ajustarse al arquetipo de la manera más precisa; tal es la noción patriarcal del etemo femenino (cuyos adjetivos son el ser dulce, suave, sentimental, superficial, frágil, dependiente, maternal, coqueta, voluble, sacrificada, envidiosa, etc., Sánchez, 1986) al cual las mujeres, mediante un acto de adscripción a tales esquemas de interpretación, quedan integradas a su dis positivo de reproducción.

La existencia de las mujeres, al no ser personal ni tendiente a la individuación, no merece ser denominada subjetividad en sentido estricto; es to es, lo que en la opinión de Celia Amorós (1992), queda conjurado por la filosofía de la modernidad, la 'otredad' como "ideologema misógino" que recorre tanto el registro ontológico como el ético. La mujer tem ina siendo definida como lo inesencial y lo relativo, solamente por decisión del hombre, del filósofo.

Tal relatividad se amplía a la imposibilidad de pensar a la mujer en sí misma, sino en cuanto a su relación con un otro; es decir, en cuanto esposa, hija, pero principalmente en cuanto madre; la condición femenina encuentra su principal alternativa de dignificación en la matemidad. 
La heteronom ía como es tatuto ético atribuido a la mujer

La estética, más que la lógica ha sido el criterio ordenador de la conducta femenina, propuesto por el pensamiento filosófico modemo; desde este punto de vista, la mujer resulta un ser incapacitado para la actitud ilustrada por excelencia, la autonomía.

Tal perspectiva encuentra nuevos desarrollos contemporáneos en la psicología: los sesgos de género que compartan las teorías psicológicas sobre el desarrollo moral, conducen a postular la inferioridad moral femenina. Bien porque el punto de vista falocrático psicoanalítico indica que la evidencia de la castración en el caso de la niña -y no la am enaza- es la condición des de la cual la mujer construye un superyó más débil que sepulta pero no supera la condición edípica, aplazando su realización -a través de una ecuación dinámica- dentro del matrimonio como relación edípica paradigmática- consiguiendo el pene de su hijo varón. (Freud, 1924).

También porque desde el punto de vista estructuralista genético, Kohlberg, tal como lo muestra Gilligan (1982) trabajando con muchachos varones construye una teoría del desarrollo genético del juicio sobre lo justo, que al ser aplicada a la valoración del desarrollo moral individual de las mujeres, atribuye a éstas la tendencia a fijarse en un estadio de desarrollo intermedio (de moralidad convencional, regida por lo socialmente sancionado), consiguiendo el ideologema misógino un nuevo refuerzo, que lleva a que investigadores como Kohlberg, decidan concluir que las mujeres parecen tener un desarrollo moral insuficiente, antes que preguntars e si la insuficiencia está en la teoría.

Lo más serio de este refinamiento es que su fachada cientificista, anclada en la pretensión de una neutralidad valorativa, desideologiza los planteamientos inequitativos para la condición humana de la mitad de la especie, poniéndolos como un azar de la naturaleza, el resultado del destino cruel e inexorable de las mujeres. La pretensión de universalidad de los planteamientos de la ilus tración fracasa precisamente en sus sesgos de género, ya que al tiempo que pretende generar las condiciones ontológicas para la igualdad, despoja a la mitad del género humano, también ontológicamente, de tales condiciones. No obstante, aquí quedan fundamentadas la comprensión ideologizada e inequitativa de las diferencias entre los géneros.

Lo anterior es tan sólo un ejemplo del enome esfuerzo de la teoría feminista crítica contemporánea por visibilizar y desnaturalizar las políticas de la identidad construidas en occidente. De alguna manera podríamos decir que su agenda ha estado centrada en la deconstrucción de las posiciones de sujeto históricamente reificadas para la mujer y lo femenino. Se trataría del comienzo de un largo camino de liberación, el cual apenas está iniciando el proceso reconstructivo mediante la consolidación de unas prácticas de liberación, las cuales enfrentan como se verá más adelante, múltiples paradojas. Las filósofas asumen la posición 
crítica de poner cuestión los fenómenos de dominación de las mujeres y por ende de la subjetividad.

Las tensiones de una filosofía feminista

Son múltiples las tensiones que enfrenta una epistemología feminista:

La pregunta por la legitimidad de teorías feministas

Tal pregunta debe ser respondida tanto des de el punto de vista científico, como desde el punto de vista ético político. Des de el punto de vista científico, afirmar la existencia de una forma de ser femenino conduce a esencializar diferencias que de otro lado se han postulado como construcciones sociales de carácter histórico: ¿si no admitimos ningún tipo de es encialismo en la definición de lo femenino, de una forma de ser mujer, cómo aceptar entonces un punto de vista y una ciencia femenina?

Una respuesta a este complicado dilema se encuentra en la obra de la Celia Amorós, cuando afirma: "Es el varón quien ha inventado nuestra diferencia. Lo único que podríamos reivindicar es lo que nosotras hemos hecho de ese invento, lo cual no es nada fácil de determinar, pero es lo único en lo que podemos identificarnos como seres con capacidad de trascendencia que no han vivido, a su vez, pasivamente, el estatuto de pasividad a que se les ha condenado". (1980:137).

Aunque como lo señala Celia Amorós (1980), tampoco el feminismo de la igualdad nos libera de paradojas semejantes, y de lo que se trata es de as umir con libertad y entereza la búsqueda de altemativas de identidad des de el ejercicio de sí-mismas y no de proponer discursos vacíos de realidad y de experiencia concreta, postulados como ideales de identidad y de vida feministas y/o para las mujeres, la aceptación de la construcción histórico-social de los géneros no nos remite necesariamente a un discurs o sobre la igualdad (que no sobre la equidad).

Es innegable que la socialización de género ha desarrollado unos roles y unas capacidades diferenciales, al tiempo que ha creado unos espacios en los cuales las mujeres han construido su propia subcultura y aquí la carencia se vuelve virtud. Las subculturas de la socialización femenina han acumulado unas prácticas de resistencia (Bellucci, 1992), las cuales, al tiempo que asimilan los dispositivos del control patriarcal, generan espacios para la construcción de su propia autonomía y emancipación. Tales prácticas pasan por una toma de conciencia sobre el poder del lenguaje en el proceso de socialización, lo cual ha posibilitado que, al entregarlo, no haga de él una transmisión neutral, sino mediada -interesada- por sus as piraciones de cambio cultural (Schmukler, 1982).

Así pues, es posible afirmar que actualmente existe un punto de vista ético femenino, entendiendo por ello el que las mujeres -en su subcultura- han acumulado a lo largo de la historia un saber sobre el ser nutricio, el cuidado 
mutuo, la solidaridad y la capacidad afiliativa, el cual sin ser consustancial o naturalmente femenino, sí constituye la contribución femenina a la construcción de una cultura alternativa. Los valores o la ética femenina están llamados a elevarse al estatuto universal que de suyo les corresponde en la construcción de nuevas utopías (Estrada, 1993).

Es también desde el principio ético de la igualdad postulado por los discursos éticos ilustrados, que el punto de vista femenino le señala los límites a la universalidad de la propuesta: tales discursos han justificado a su vez la doble moral y la existencia de una jerarquía entre los géneros que ha sustentado históricamente la subordinación de uno con respecto al otro. De hecho, el propio Kohlberg, en respuesta a las críticas, señaló cómo su trabajo se centró en la reconstrucción ontogenética del juicio sobre lo justo y cómo el juicio moral y la acción moral son necesariamente complementarios, tanto como lo son la orientación hacia la justicia y hacia el respeto y el cuidado mutuos. (Levin, Kohlberg y Hewer, 1985).

Así pues, los estudios de la mujer y de Género, se justifican, al menos en un momento histórico como el nuestro, en el cual aún nos falta mucho por comprender de la especificidad de la subcultura femenina y de su potencialidad de resistencia, frente al caos y la barbarie social en que estamos sumidas/sumidos.

Desde el punto de vista estratégico parece necesario que el movimiento de mujeres sea apoyado por los 'Estudios de la Mujer' y viceversa, de forma que tal movimiento contribuya al señalamiento de las realidades concretas de las mujeres, de las cuales debe partir y no apartarse una ciencia comprometida con la liberación de la condición del ser mujer en América Latina. Igualmente se acepta que los movimientos sociales en sí mismos comportan un potencial liberador para la ciencia, que ella debe aprovechar.

Este apoyo recíproco debe redundar en formas de organización para la lucha contra todas las formas de discriminación y en la aclaración de un espacio para la evaluación y la transformación de las disposiciones de género existentes (Flax, 1990), donde la intención de construirse como sujetos y resingnificar lo femenino sean posibles. (Bonders.d.).

Tanto los 'Estudios de la Mujer', desarrollados en espacios especializados, como los 'Estudios de Género' agenciados desde las instituciones académicas tradicionales, parecen necesarios para la instauración de un nuevo orden sociocultural, donde más mujeres y hombres acepten los sesgos de género que ha comportado la ciencia ilustrada de la modernidad y empleen la categoría de género como una posibilidad de ampliar la mirada y la gama de matices -así captados- en la realidad social.

Las diferentes perspectivas al interior de los 'Estudios de Mujer'.

Sandra Harding (1990.) recoge en dos, las fuerzas más características que se dan -como paradigmas o estrategias justificatorias - al interior de los 'Estudios de la 
Mujer'. Las denomina: empirismo femenino y teorías desde el punto de vista fem inis ta respectivamente.

La diferencia básica entre las dos se encuentra en que, mientras el empirismo feminista afirma que es posible eliminar los sesgos sexistas de los paradigmas científicos tradicionales, mediante una adhesión más estricta a sus propias reglas y cánones metodológicos, las teorías del punto de vista sostiene que es este tipo de investigación la que socava la distorsión de las ciencias androcéntricas occidentales, tradicionalmente negadoras de los matices sociales.

Mientras la primera es más conservadora y podríamos decir, trabaja 'desde adentro', la segunda es más receptiva al papel de los movimientos sociales y políticos en el desarrollo mismo de la comprensión: trabaja 'desde afuera' a partir de las preguntas que surgen en la perspectiva de las actividades de las mujeres. La mayoría de las teóricas que discuten es ta tensión, están de acuerdo en señalar que ambas pers pectivas son necesarias en el momento actual.

Sin embargo, para Sandra Harding (1990), ambas posiciones se vienen desplazando fuera del paradigma ilustrado de ciencia, para ubicarse en lo que ella denomina un 'posmodernismo feminista'; sin embargo, también desde el socioconstruccionismo de Gergen (1985), se reporta tal desplazamiento, cuando se señala cómo, muchas pensadoras feministas han atacado el punto de vista empiricista en ciencia y han adoptado una perspectiva interpretativa para el tratamiento de los datos empíricos, lo cual las acerca a un paradigma donde la idea de la construcción social de la realidad encuentra sus tento.

El género como dispositivo para la producción de la subjetividad

...un dispositivo es una especie de ovillo o madeja, un conjunto multilineal. Está compuesto de líneas de diferente naturaleza y esas líneas del dispositivo no abarcan ni rodean sistemas cada uno de los cuales sería homogéneo por su cuenta (el objeto, el sujeto, el lenguaje), sino que siguen direcciones diferentes, forman procesos siempre en desequilibrio y esas líneas tanto se acercan unas a otras como se alejan unas de otras. Cada línea está quebrada y sometida a variaciones de dirección (bifurcada, ahorquillada), sometida a derivaciones.

Deleuze, 1989:155

Ya en otro lugar (Estrada y otras 2003; Estrada, 2002), abordé la noción de dispositivo cultural como la es tructuración compleja de elementos propios de por lo menos tres ordenes diversos: (a) el orden de la imaginería cultural; caracterizado por representaciones y creencias fuertemente arraigadas en culturas locales específicas, (b) el orden de la acción y la interacción; en el cual se configuran patrones que se activan en las dinámicas sociales concretas dando origen a, (c) estrategias de autorregulación de la propia subjetividad. La articulación de los mecanismos pertenecientes a cada uno de tales niveles es lo que da origen a un dispositivo particular. Ahora bien, los dispositivos culturales pueden funcionar de 
manera naturalizada y no consciente en las culturas locales, o bien, de manera intencionada y reflexiva.

Los dispositivos son mediaciones sociales e institucionales complejas con un alto potencial estructurante de la subjetividad, ya que circulan libremente en los distintos escenarios sociales y de la vida privada, articulando mecanismos y procesos de órdenes diversos. Comprender el funcionamiento específico de los dispositivos para la producción de la subjetividad requiere "...en cada caso levantar un mapa, cartografiar, recorrer tierras desconocidas..." (Deleuze, 1989: 155) que atraviesen y busquen los entrecruzamientos entre fragmentos de distintos órdenes. En tal sentido, uno de los principales retos contemporáneos se relaciona con la reconstrucción del sujeto en su relación con las instituciones, con las prácticas sociales y con los procesos subjetivos, a partir de la comprensión de la experiencia como prácticas concretas de subjetivación (de Lauretis, 1989).

En efecto, el género como categoría crítica contribuye a poner en evidencia los a priori históricos de los discursos sobre el sujeto, particulamente aquellos que sostienen la 'episteme de lo mismo' (Fernández, 1993), la cual efectúa para las ciencias sociales la razón totalizante y universal de la filosofía moderna.

Instaurada en la lógica de la identidad, la 'episteme de lo mismo' no puede captar la diferencia -la otredad-, más que como lo contingente o desviado con referencia a lo mismo; lo cual, al no poder pensarse como lo otro, se transforma en lo único (Fernández, 1993).

Desprovis to de todo sesgo es encialista, el género aparece como una categoría relacional mediada por dispositivos de poder (Scott, 1996). Es decir, en los intercambios sociales se activan acuerdos históricamente construidos para la interpretación de la subjetividad femenina y masculina. Interpretación que en sentido psicológico, no está determinada por el sexo de los actores, sino por el contexto discursivo de las interacciones, particulamente el poder y el estatus (Bohan, 1997), y abandona la binariedad que se atribuye al sexo en sentido biológico concebido como causa del género en tanto identidad psicosocial.

Comprendida así, la perspectiva de género es una categoría analítica para abordar las instituciones y las prácticas sociales que potencialmente tienen el poder cultural, no sólo para pautar las relaciones entre hombres y mujeres, sino para apoyar su construcción y desarrollo diferencial. Desde este punto de vista, los seres humanos, mediante su participación activa, as umen o controvierten las reglas y códigos que pautan las relaciones entre los géneros y su estatus diferencial, subyacentes o implícitas en las culturas locales.

Una mirada con lente de género pemite hacer visibles distintas posiciones de sujeto que encarnan expresiones de la masculinidad y la feminidad en un contexto relacional, cuya estructura profunda, está nutrida no sólo o directamente por el contexto patriarcal, sino también por las distintas narrativas comunitarias que desde múltiples fuentes entran, circulan y se activan en las interacciones sociales y las prácticas institucionales y cuyo trasfondo es moral, en cuanto genera una 
estructura valorativa y jerarquizada de la diferencia, desde la cual se prescriben competencias e incompetencias diferenciales

Penetrar la diferencia implica el reconocimiento y la búsqueda explícita de la interdependencia entre los sistemas de significado individuales y los sociales o culturales. Esto a su vez, exige que partiendo de la experiencia de los distintos actores en sus contextos particulares, ésta se reconstruya en un nivel de análisis más amplio dentro de marcos culturales y sociales de poder (Henwood y Pidgeon, 1995).

En el ámbito subjetivo, la construcción del géneros es el resultado de inscripción en el cuerpo de unos discursos históricamente construidos y culturalmente legitimados sobre la sexualidad y las identidades sexuales, as í como los procesos de resistencia que desde distintas posiciones de sujeto tienen lugar, frente a las narrativas oficiales. Desde este punto de vis ta la identidad de género es la información cultural de la materia, proceso mediante el cual deviene materialidad. Este punto de vis ta sobre el género ofrece la ventaja de integrar de manera muy precisa nociones culturales, históricas, sociológicas y psicológicas en la construcción de la noción de género como una categoría estructural relacional inscrita en el microjuego cotidiano de los poderes para comprender la producción de distintas posiciones de sujeto (Butler 1987, Estrada, 1997).

Judith Butler (1987) logra extraer las implicaciones de la concepción foucaultiana de la inscripción de discursos históricos en el cuerpo, haciendo énfasis en la comprensión de una identidad incardinada (encamada). En efecto, cuando Foucault (1977) afirma que el poder atraviesa los cuerpos, no explica cómo tiene lugar ese proceso de subjetivación. Entre tanto, la distinción elaborada por Butler (1993) entre materia y materialidad mues tra cómo el cuerpo mismo, pasa de ser materia pura para constituirse en materialidad, en corporealidad, en la medida en que es informado por la serie de discursos históricos. En este sentido, el género es la corporalidad.

Así pues, la unidad del sujeto es puesta bajo sospecha mediante la distinción entre sexo y género, en la medida en que éste último se entiende como una interpretación múltiple del primero: "Llevada hasta su límite lógico la distinción sexo/género indica una discontinuidad radical entre los cuerpos sexuados y los géneros construidos" (Butler, 1990a: 39). Se abandona la idea de una concepción mimética entre el sexo y el género.

Lo anterior, conduce a otro problema: ¿es el sexo una estructura natural?, o, ¿es, por el contrario, la interpretación que de unos marcadores biológicos han hecho las distintas ciencias biológicas y de la salud? Es interesante traer aquí los estudios críticos sobre las metáforas del conocimiento biológico que muestran la asociación de la estructura biológica femenina con la pasividad y la masculina con la actividad (Gergen, 1990). 
Lo anterior nos lleva a señalar que si los hechos biológicos, como los de cualquier otra ciencia, se producen en los discursos científicos, la categoría sexo es tan socialmente construida como la de género y la distinción entre las dos, no existe como tal. La binariedad del género parece servir a las políticas de la identidad que confiscaron a partir de siglo XVII la sexualidad para la reproducción y la conyugalidad, generando un dispositivo de subjetivación regulada por una matriz heterosexual y la naturaleza sexuada deja de ser una categoría prediscursiva neutral en la cual actuaría la cultura (Butler, 1990a). Por este camino, hombre y mujer llegar a comprenderse como momentos de la historia de la construcción de los géneros (Butler, 1987).

Al hacer evidente el hecho de que la noción de género has ta ahora vigente ha aceptado y asumido acríticamente la idea de que la aparente bipolaridad biológica de los sexos tiene su correspondiente psíquica, da paso a la proliferación de los géneros/cuerpos y a la deconstrucción de la concepción bipolar de los mismos. No se trata tanto de una concepción andrógina del sujeto, cuanto de poner en cuestión la matriz heterosexual como el único campo legítimo para la subjetivación, la construcción del deseo y la identidad.

Una de las mayores tensiones internas del feminismo está en la prioridad estratégica de la categoría mujer para el accionar político, al tiempo que su uso tiende a esencializar el género y a reproducir el sujeto mujer subordinado socialmente y la exclusión o la estigmatización de otras posiciones de sujetos que no se adecuan a la matriz heterosexual.

Se da paso así a una concepción preformativa del género y de la subjetividad que busca desarrollar teóricamente las implicaciones de asumir que éstos se fabrican mediante una serie de actos repetitivos de estilización del cuerpo basados en el género entendido como interpretación cultural. Esta indagación parte de precisar que lo que hemos entendido como rasgos intemos de nosotro/as mismo/as, son más bien anticipaciones que producimos mediante ciertos actos corporales (Butler, 1990a). Tal vez aquí hay una enome convergencia con la propuesta de Foucault (1982) en el sentido de que el sujeto no es una sustancia, sino forma, la identidad más que configurar un núcleo interno del si mismo/a, es exterioridad pura: el género, no es más que una fantasía inscrita en la superficie del cuerpo (Butler, 1990b).

¿Cuál es el lugar de filosofía feminista en el debate contemporáneo sobre el sujeto?

Son muchos los investigadores externos al campo de la filosofía feminista que reconocen explícitamente la función crítica que ésta ha cumplido en la puesta en cuestión de la concepción moderna y occidental de sujeto, tanto en sus implicaciones epistemológicas, como éticas y sociales.

Transformación de las metáforas sobre el sujeto 
La máquina, el organismo vivo y el ordenador, hacen parte de las metáforas que se han anclado en nuestra imaginería cultural, con el trabajo de la filosofía y de las ciencias sociales, como modelos a partir de los cuales narramos lo psíquico, el yo, el si-mismo/a. El sujeto individual y sus prácticas individualistas aferradas a una noción de autonomía autocontenida, son uno de los productos más refinados de la modemidad, el cual convive en todo caso con los retazos de una concepción romántica de la autenticidad y el conocimiento de si.

La saturación cultural que experimenta el yo contemporáneo, se recrea en los distintos ámbitos de la cultura incluyendo la academia. El yo se ha tornado una hiperealidad (Gergen, 1991) a medida que sus múltiples y sucesivas narraciones en occidente han hecho densa y confusa su configuración de significado y el mundo globalizado efectúa una transformación del tiempo y el espacio. La exacerbación del individualismo cuyo núcleo es un yo autocontenido, está dando señales inequívocas de su potencial de aniquilación.

Los proyectos en curso orientados a la deconstrucción del yo, requieren una vigilancia que presione el desarrollo de propuestas teóricas ancladas sobre la diferencia, incluyentes de lo otro y orientadas a la búsqueda y defensa de prácticas liberadoras del sujeto, de juegos de poder que acuerden la no dominación. Sin duda, el desarrollo de tales prácticas, requiere, a su vez, de teorías performativas de la psique y del sujeto que partan de un cuestionamiento sobre la objetivación los patrones sociales, suceso que ha ocurrido gracias a la objetivación de nuestras prácticas sociales y disciplinares

Los desarrollos teóricos sobre la existencia de una lógica moral relacional que no as piran a la autonomía autocontenida de la lógica moral formal, como foma superior de la subjetividad, constituyen un patrón de transformación del sujeto individualista que la filosofía feminista está en condiciones de contribuir a aclimatar en un horizonte de transfomación cultural, toda vez que cuenta con un saber acumulado sobre las prácticas relaciones de cuidado y solidarias con la vida.

La crítica cultural y la cons trucción de nuevos mundos

La exploración de las formas de resistencia presentes en la cultura, son sin duda una contribución importante a la tarea de exploración de las prácticas sociales, conversaciones y descripciones humanas acerca de si-mismo/as y de la vida social cuyos téminos sean relacionales más que individuales, tanto en las culturas locales, como en el mundo globalizado, con potencial para fortalecer una transfomación de la cultura y del yo.

El yo comienza a reconocerse como las multivoces -femeninas y masculinasde la narración microsocial y de los relatos personales; el relato de la primera persona en la trama de las narraciones sociales que lo co-constituyen. Aquí las metáforas relacionales se desplazan desde la red hasta la comunidad políticamente comprometida. 


\section{BIBLIOGRAFIA CITADA}

Bohan, Janis (1997). 'Regarding gender: essentialism, constructionism, and feminism psychology'. En: Gergen, Mary y Sara Davis (eds.) Toward new psychology of gender. A reader. Estados Unidos, Routledge,31-48.

Butler, Judith (1993). Bodies that matter. On the discursive limits of "sex". Estados Unidos, Routledge.

(1990a). El género en disputa. El feminismo y la subversión de la identidad. México, Paidós.

(1990b). 'Gender Trouble. Feminist Theory, and Psychoanalytic Discourse'. En: Nicholson, Linda (ed.) Feminsm/Postmodernism. Estados Unidos, Routledge.

(1987). 'Variaciones sobre sexo y género. Beauvoir, Wittig y Foucault' En: Benhabib, Seyla y Drucilla Comella. Teoría feminsta y teoría crítica. España, Alfons el Magnanim.

De Lauretis, Teresa (1989). 'La es encia del triángulo, o tomarse en serio el riego del esencialismo: teoría feminista en Italia, Ios E.U.A. y Gran Bretaña'. Debate Feminista. Añol, Vol. 2, sept, 1990, pp. 77-115.

Deleuze, Gilles (1989). ‘¿Qué es un dispositivo?'. En: Balbier, E., y otros (1989). Michel Foucault, filós ofo. España, Gedisa.

Estrada, Angela María, Carolina Ibarra y Estefanía Sarmiento. 'Regulación y control de la subjetividad y la vida privada en el contexto del conflicto armado colombiano'. En prensa.

(2001). "Los fragmentos del calidoscopio. Una propuesta teórica metodológica para el análisis cualitativo de las relaciones de género en la escuela". Revista Nómadas, No, 14, abril, p. 10-23.

(1997). "Los estudios de género en Colombia: entre los límites y las posibilidades". Revista Nómadas, No, 6, marzo, p. 35-54.

Fernández, Ana María. 'La bella diferencia'. En: idem. La mujer de la ilusión. Pactos y contratos entre hombres y mujeres. Argentina, Paidós, 1993.

Foucault, Michel (1976). 'Nosotros los victorianos'. En: Idem. Historia de la sexualidad 1. la voluntad de saber. México, Siglo XXI, 1977.

(1977) . 'Las relaciones de poder penetran en los cuerpos'. En: Idem. Microfísica del poder. España, la Piqueta. 
(1984). 'La ética del cuidado de uno mismo como práctica de la libertad'. Entrevista con M. Foucault. En: Idem. Hermenéutica del sujeto. Madrid, La Piqueta, 1994.

Freud, Sigmund. (1924). 'El Sepultamiento del Complejo de Edipo'. Obras. Tomo XIX. Buenos Aires, Amorrortu, 1984. Gergen, Kenneth (1991). El yo saturado. España, Paidós.

(1990). 'Metaphor, metatheory and the social world'. En: Leary, D. Metaphors in the history of psychology. Estados Unidos, Cambridge University Press.

(1985) 'The Social Constructionist Movement in Modern Psychology'. American Psychologist. 40, 3, 266-275.

Gilligan, Carol. (1982). La Moral y la Teoría, Psicología del Desarrollo Femenino. México, F.C.E., 1985.

Harding, Sandra (1990). 'Feminism, Science and the Anti-Enlightenment Critiques'. En: Nicholson, Linda. Feminism/Postmodernism, Estados Unidos, Routledge.

Henwood, Karen \& Nick Pidgeon, (1995). 'Grounded theory and psychological research'. The psychologist. Vol. 8, No. 3, march 1995, p.115-118.

Loraux, 1991

MacNamee, Sheila y Kenneth Gergen (1999). Relational responsability. Resources for sustainable dialogue. Estados Unidos, Sage.

Scott, Joan (1986). "El género: una categoría útil para el análisis histórico". En: Lamas, Marta (comp.) (1996). El género: la construcción cultural de la diferencia sexual. México, UNAM. 